\title{
Atomic hydrogen interactions with small polycyclic aromatic hydrocarbons cations ${ }^{\star}$
}

\author{
Thomas Schlathölter ${ }^{1, a}$, Yahia Mostafa ${ }^{1}$, Amber Kamman ${ }^{1}$, Arnold Dongelmans ${ }^{1}$, Yann Arribard ${ }^{1}$, \\ Stephanie Cazaux $^{2,3}$, and Ronnie Hoekstra ${ }^{1}$ \\ 1 Zernike Institute for Advanced Materials, University of Groningen, Nijenborgh 4, 9747 AG Groningen, The Netherlands \\ 2 University of Leiden, P.O. Box 9513, 2300 RA Leiden, The Netherlands \\ 3 Faculty of Aerospace Engineering, Delft University of Technology, Kluyverweg 1, 2629 HS Delft, The Netherlands
}

Received 24 February 2020 / Received in final form 6 April 2020

Published online 11 June 2020

(C) The Author(s) 2020. This article is published with open access at Springerlink.com

\begin{abstract}
When exposed to a thermal beam of hydrogen atoms, gas-phase coronene cations $\mathrm{C}_{24} \mathrm{H}_{12}^{+}$can be sequentially hydrogenated. This process is accompanied by a gradual transition of the electronic structure from aromatic to aliphatic. The planar very stable coronene structure transforms into the significantly weaker corrugated structure, typical for aliphatic molecules. In this study, we have investigated the hydrogenation of 5 smaller polycyclic aromatic hydrocarbon cations using a combination of radiofrequency ion trapping with time-of-flight mass spectrometry. Anthracene $\left(\mathrm{C}_{14} \mathrm{H}_{10}^{+}\right)$, pyrene $\left(\mathrm{C}_{16} \mathrm{H}_{10}^{+}\right)$, triphenylene $\left(\mathrm{C}_{18} \mathrm{H}_{12}^{+}\right)$, tetracene $\left(\mathrm{C}_{18} \mathrm{H}_{12}^{+}\right)$and 8-9-benzofluoranthene $\left(\mathrm{C}_{20} \mathrm{H}_{12}^{+}\right)$only cover a small mass range, but differ in carbon/hydrogen ratio, number of outer-edge sites and overall structure. We have observed qualitatively similar initial hydrogenation patterns for all 5 molecular ions, with odd hydrogenation states being dominant. Strong quantitative differences in hydrogenation and in attachment-induced fragmentation were found. For the case of pyrene cations, we have also investigated exposure to atomic D. Clear lines of evidence for $\mathrm{HD} / \mathrm{D}_{2}$ abstraction reactions of Eley-Rideal type were found, as previously observed for coronene cations.
\end{abstract}

\section{Introduction}

Polycylic aromatic hydrocarbons (PAHs) are most certainly the carriers of the aromatic infrared bands emitted by many galactic and extragalactic sources $[1,2]$. In the interstellar medium (ISM), PAHs can be present in neutral form or as ions [3,4]. PAHs are of great astrochemical relevance because they provide large surfaces for chemical reactions to occur, while such reactions are inefficient in the gas phase [5]. In a pioneering study, Snow et al. have studied the reactivity of small PAH cations such as $\mathrm{C}_{16} \mathrm{H}_{10}^{+}$ (pyrene) and found evidence for atomic hydrogen attachment, suggesting that hydrogenated $\mathrm{PAH}$ ions are abundant species in the ISM [6]. Rauls and Hornekær have later predicted that $\mathrm{H}_{2}$ molecules could be formed in an EleyRideal process from two hydrogen atoms co-adsorbed on a neutral PAH under conditions typical of the ISM [7]. Successive hydrogen attachment and $\mathrm{H}_{2}$ formation was first experimentally confirmed for neutral coronene $\left(\mathrm{C}_{24} \mathrm{H}_{12}\right)$ thin films on a solid substrate [8].

* Contribution to the Topical Issue "Atomic Cluster Collisions (2019)", edited by Alexey Verkhovtsev, Pablo de Vera, Nigel J. Mason, Andrey V. Solov'yov.

a e-mail: t.a.schlatholter@rug.nl
Our group has tackled hydrogen attachment on PAHs from a gas-phase perspective. We have exposed $\mathrm{C}_{24} \mathrm{H}_{12}^{+}$ (coronene) cations held in an ion trap to a thermal beam of atomic hydrogen and found evidence for attachment of multiple $\mathrm{H}$ atoms at room temperature [9]. Generally, $\mathrm{H}$ attachment to coronene (cor) cations can be described by sequences of the reaction

$$
[\text { cor }+s \mathrm{H}]^{+}+\mathrm{H} \rightarrow[\operatorname{cor}+(s+1) \mathrm{H}]^{+}, \quad \Delta M=+1
$$

where $s$ represents the number of extra $\mathrm{H}$ atoms attached. Each of these reactions increases the mass $M$ by $1 \mathrm{Da}$. In a combined experimental and theoretical study we could show that $\mathrm{C}_{24} \mathrm{H}_{12}^{+}$hydrogenation at $T=300 \mathrm{~K}$ is following a well-defined sequence of hydrogenation sites [10] that has later been confirmed by means of infrared spectroscopy [11]. The attachment barriers almost vanish for radicals with even numbers of attached $\mathrm{H}$ atoms and as a consequence, cations with odd $s$ are observed at much higher intensities than cations with even $s$. Hydrogenation states with $s=5,11$ and 17 are particularly prominent due to their relatively high binding energies combined with a high barrier for the next hydrogenation to occur. Very recently, Jensen and coworkers have also identified particularly stable configurations with $s=2,4,8,10,14,18$, and 24 for supported thin films of neutral coronene [12] (Note that in neutral coronene, systems with an odd $s$ 


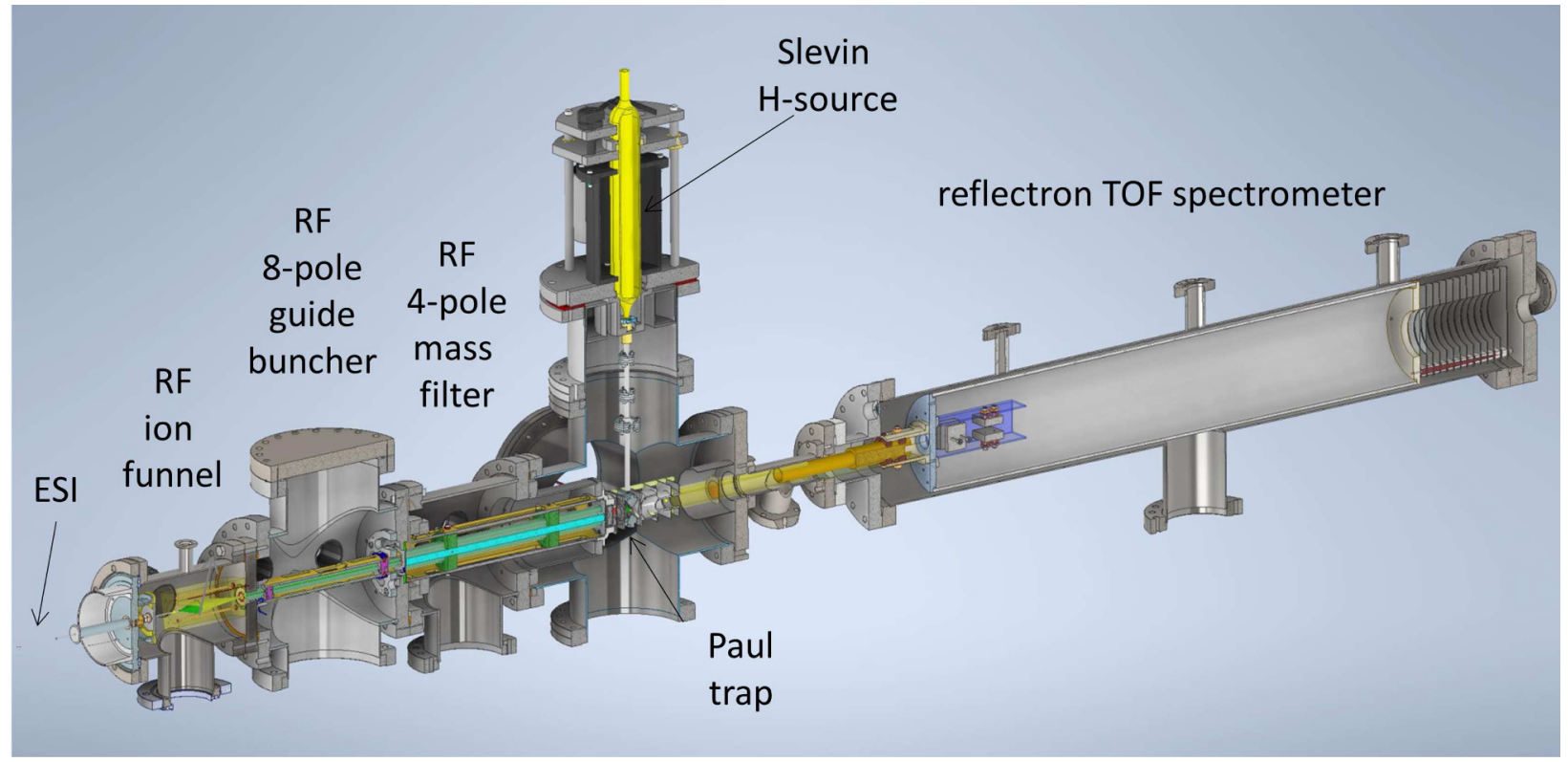

Fig. 1. Sketch of the experimental setup, displaying from left to right the electrospray ion source, the RF ion funnel, the octopole RF ion guide and buncher, the quadrupole mass filter, the RF ion trap and the reflectron TOF system. The atomic hydrogen source is located right above the Paul trap.

have radical character and therefore even $s$ will dominate the mass spectra).

Hydrogen attachment competes with hydrogen abstraction processes of the Eley-Rideal type

$$
[\operatorname{cor}+s \mathrm{H}]^{+}+\mathrm{H} \rightarrow[\operatorname{cor}+(s-1) \mathrm{H}]^{+}+\mathrm{H}_{2}, \quad \Delta M=-1 .
$$

Abstraction processes can occur for cationic and neutral coronene and have been first experimentally confirmed by Menella et al. for supported coronene thin films (neutral coronene), albeit with a cross section of $0.06 \AA^{2}$ which is about 20 times smaller than the corresponding cross section for $\mathrm{H}$ attachment $1.1 \AA^{2}[13]$. We have recently studied abstraction processes on gas-phase coronene cations using atomic deuterium and observed an order of magnitude larger abstraction cross sections of about $0.45 \AA^{2}$ for this system [14]. Such large cross sections of cationic coronene compared to neutral coronene, could render this process relevant for $\mathrm{H}_{2}$ formation in the ISM, and make cationic PAHs important interstellar catalysts.

Most experimental studies on hydrogenation have until now focused on coronene $\mathrm{C}_{24} \mathrm{H}_{12}$, either neutral or as a cation. Campisi et al. [15] have very recently shown for (neutral) PAH thin films, hydrogenation in the linear catacondensed $\mathrm{PAH}$ pentacene begins at the $\mathrm{C}-\mathrm{H}$ complex in the central ring. This site has no neighboring $\mathrm{C}-\mathrm{H}$ group and therefore differs from the first hydrogenation site in coronene. In this article, we present experimental results on the hydrogenation of 5 smaller PAH cations; anthracene, pyrene, triphenylene, tetracene and 8-9-benzofluoranthene which have masses in the range from $178 \mathrm{Da}$ up to $252 \mathrm{Da}$. The molecular structures of these molecules however (see Fig. 2), are very different and range from linear (anthracene, tetracene) over compact (pyrene) to very open (triphenylene). 8-9-fluoranthene even contains a 5-membered ring. It will be discussed how the character of the different potential H-attachment sites influences hydrogenation. For the case of pyrene, we will also present data on atomic deuterium attachment that clearly demonstrates the role of Eley-Rideal abstraction reactions.

\section{Experiment}

A sketch of the apparatus is shown in Figure 1. The experiment has been described in previous work [16]. Briefly, we have employed electrospray ionization to produce radical cations of anthracene, pyrene, triphenylene, tetracene and 8-9-benzofluoranthene (all PAHs had at least 99\% purity, Sigma Aldrich). A saturated solution of the respective PAH in methanol was prepared and diluted with an appropriate amount of methanol, with volume ratios ranging from 2:1 (coronene) to about 1:40 (all smaller PAHs under study). Volumetric $5 \%$ of a $10 \mathrm{mM}$ methanol diluted solution of silver nitrate $\left(\mathrm{Ag}^{+} \mathrm{NO}_{3}^{-}\right)$was added to the obtained PAH solution, to facilitate formation of radical PAH cations in solution by charge transfer to solvated $\mathrm{Ag}^{+}$ions. The phase-space of the electrosprayed beam of PAH cations was then compressed in a radiofrequency (RF) ion funnel from where they were transported into an octopole RF ion-guide that was also used as a beam buncher. Bunches of ions were then passed through a quadrupole mass filter to isolate the desired precursor ion from beam contamination. Facilitated by a pulse of $\mathrm{He}$ buffer gas injected right into the 3D RF trap (Paul type), the trap was filled with ions from a bunch of precursor ions. 
A beam of atomic hydrogen was produced in a Slevintype $\mathrm{RF}$ discharge source that operated at a frequency of $27 \mathrm{MHz}[9,17,18]$. Teflon tubing was used to guide atomic hydrogen towards the ion-trap and to thermalize the atom beam to about $300 \mathrm{~K}$. The discharge source was pulsed by electronic control of the RF field. When the RF field was off, the dissociation degree within the beam dropped to zero and only molecular hydrogen could enter the ion trap. At room temperature, molecular hydrogen does not attach to PAH cations. This way, exposure to thermal atomic hydrogen could be controlled. Typical exposure times ranged from 0 to $15 \mathrm{~s}$, the latter giving rise to moderate hydrogenation under the present experimental conditions. The precise coronene-hydrogen collision rate is difficult to determine from the experimental parameters, as we operate the hydrogen source such that the base pressure in the RF-trap chamber remains unaffected in the $10^{-6}$ mbar region. For an estimated trap content of $10^{4}$ ions within a volume of $0.5 \mathrm{~mm}$ diameter and a collision cross section of $121 \AA^{2}$ [19], we estimate a collision rate of a few $100 \mathrm{~s}^{-1}$. This is in agreement with the experimental data in the sense that we observe almost full single hydrogenation after $0.1 \mathrm{~s}$ of exposure, with a $\mathrm{H}$ attachment rate that is about one order of magnitude smaller than the collision rate [14].

Eventually, the hydrogenated $\mathrm{PAH}$ cations were extracted into a time-of-flight (TOF) spectrometer. The data for the first part of the study (hydrogenation as a function of PAH size/type) were taken with a linear TOF system with a resolution $M / \Delta M \approx 300$. The $\mathrm{H}_{2}$ abstraction experiment was performed using a reflection-type TOF system with a maximum resolution $M / \Delta M \approx 2800$.

\section{Results and discussion}

\section{$3.1 \mathrm{H}$ attachment}

Figure 2 displays an overview of mass spectra for the 5 $\mathrm{PAH}$ cations under study, obtained after $10 \mathrm{~s}$ of exposure to thermal atomic hydrogen. In all spectra, the black line corresponds to the precursor ion and the red line corresponds to the hydrogenated system. In the precursor spectra, a lower intensity peak is observed at $M+1$ which is attributed to the natural abundance of ${ }^{13} \mathrm{C}$ in the prepared samples. The precursor spectra of each PAH cation have been normalized to a maximum intensity of the precursor peak of 1 . Note that for long $\mathrm{H}$-exposure times, we occasionally observe small contributions of ions, heavier than the precursor (in Fig. 2 this can be seen in the top panel). We attribute these to reactions with reactive species originating from contaminants in the plasma.

For each PAH, the precursor spectrum has been multiplied by the factor given on the respective panel, to get comparable intensities as for the hydrogenated system. For all cations, both hydrogenation (leading to masses larger the precursor cations) and fragmentation (leading to ions with masses smaller than the precursor cations) are observed. The insets always show a zoom into the hydrogenation region, with the the number of attached $\mathrm{H}$ atoms on the $x$-axis. The attachment of an $\mathrm{H}$ atom to a PAH

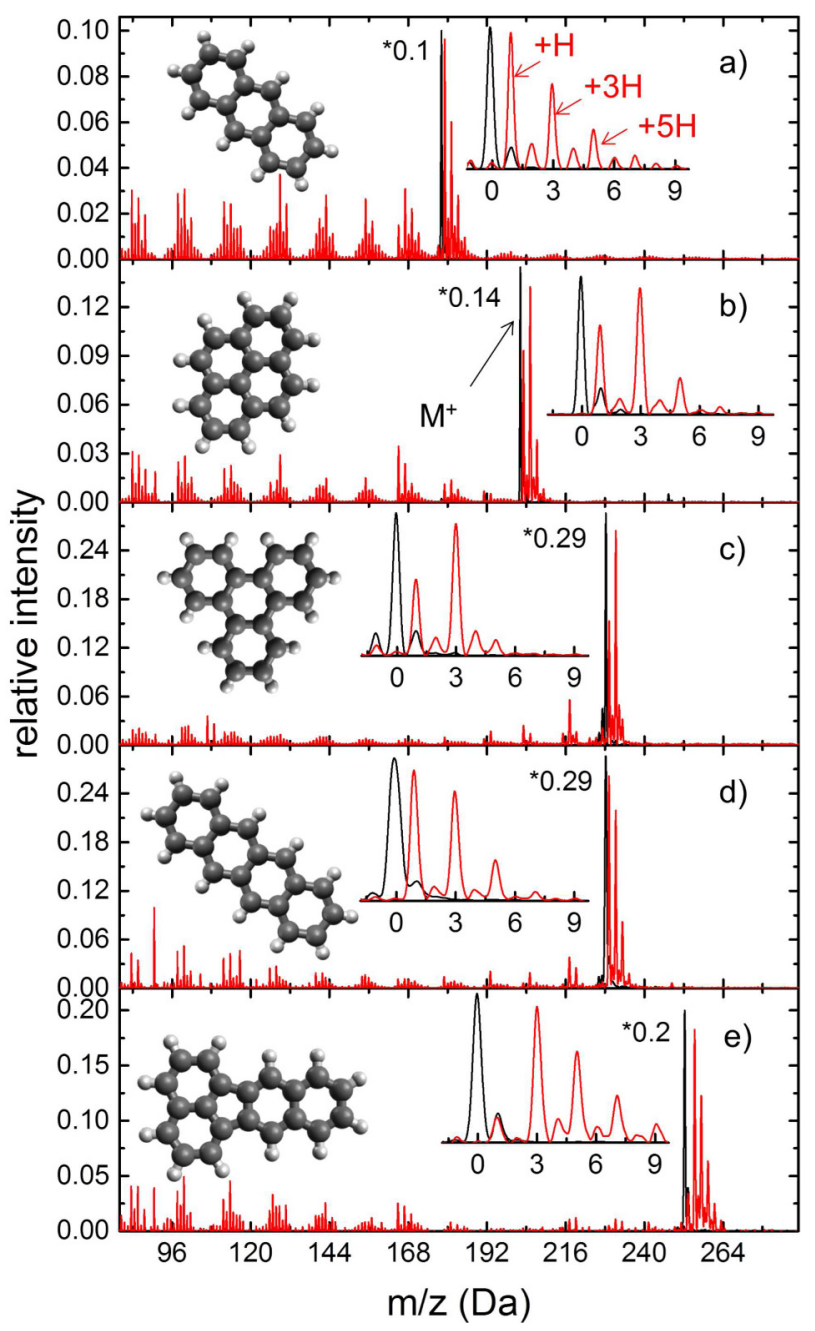

Fig. 2. Compilation of mass spectra for anthracene (a), pyrene (b), triphenylene (c), tetracene (d) and 8-9-benzofluoranthene. The black line corresponds to the mass spectrum of the respective precursor cation. The red lines show the mass spectra after $10 \mathrm{~s}$ exposure to atomic hydrogen. The insets show the highmass side of the precursor ion, i.e. the hydrogenated cations, with their mass given in number of added hydrogen atoms.

cation increases the internal (vibrational) energy of the newly formed system by the $\mathrm{H}$ binding energy. This binding energy amounts to $1.9-3.6 \mathrm{eV}$ for coronene cations [10] and is most likely in a similar range for the somewhat smaller PAH cations under study here. As a consequence, multiple hydrogen attachment can lead to fragmentation of the molecular cation, with the degree of fragmentation depending on PAH size and structure. Fragments are generally of $\mathrm{C}_{n} \mathrm{H}_{m}^{+}$type and therefore for a given $n$, usually a group of peaks with different $m$ is observed. As the cutoff of the trap is in the range of $80 \mathrm{Da}$, only groups of peaks with $n \geq 7$ are observable.

\section{Anthracene $\left(\mathrm{C}_{14} \mathrm{H}_{10}^{+}, M=178\right)$}

Figure 2a shows the results for anthracene, the smallest PAH under study. As in our previous studies for coronene 
cations, we observe clear evidence for multiple hydrogenation with the expected odd-even oscillations in peak intensity. Peaks for $s=1,3$ and 5 dominate but even $s=7$ is clearly visible. The number of available hydrogenation sites determines both, the cross section for hydrogen attachment and the maximum number of hydrogen atoms that can be attached, and it is therefore not a surprise that for anthracene (14 C atoms), less hydrogenation is observed than for coronene (24 C atoms) [20]. The maximum intensity here is observed for $s=1$, though, i.e. most cations are only singly hydrogenated. Furthermore, the relative intensity of the $s=1$ peak is only 0.1 .

The fragmentation pattern displayed in Figure $2 \mathrm{a}$ exhibits some unusual properties. First of all, fragmentation is remarkably strong with a multitude of peaks that have maximum relative intensities between 0.02 and 0.03. More importantly however, we observe groups of fragments with $n=7-13$ that all have similar intensities. It has for instance been shown by photodissociation [21], collision induced dissociation [22], electron impact ionization [23] and keV ion collisions [24,25] that for anthracene (and other PAHs) and their ions, loss of $\mathrm{H}$ and $\mathrm{C}_{2} \mathrm{H}_{2}$ are the dissociation channels with the lowest activation energies [26]. Higher excitation energies lead to more extensive fragmentation. Typical spectra at higher excitation therefore feature a strong fragment group due to $\mathrm{C}_{2} \mathrm{H}_{2}$ loss with $n=12$, weaker intensities for fragments with $n=8-11$ and strong groups of fragments with $n \leq 7$. Loss of $\mathrm{C}_{1} \mathrm{H}_{m}$ groups leading to $n=13$ fragments has been induced by knockout reactions in anthracene ${ }^{+}$collisions with $\mathrm{He}$ at center-of-mass energies around $100 \mathrm{eV}$ [27] but knock-out processes are energetically ruled out for interactions with thermal $\mathrm{H}$ atoms. Rapacioli et al. [20] have studied the fragmentation of multiply hydrogenated coronene cations for different distributions of excitation energies using a density functional based tight binding approach. Their results for a hot $(3500 \mathrm{~K})$ molecule that has not undergone any cooling (Boltzmann scenario) are closest to the mass spectrum in Figure 2a, with the sole exception that $\mathrm{C}_{1} \mathrm{H}_{m}$ is about a factor of two weaker in the calculations. From this we conclude that anthracene cations are sufficiently small that $\mathrm{H}$ attachment leads to a strong temperature increase that induces swift thermal fragmentation before de-excitation processes can occur.

Pyrene $\left(\mathrm{C}_{16} \mathrm{H}_{10}^{+}, M=202\right)$

Figure $2 \mathrm{~b}$ shows the results for the 4 ring $\mathrm{PAH}$ pyrene. This compound is the smallest peri-condensed $\mathrm{PAH}$, where three or more rings share a common carbon atom. Again, multiple hydrogenation is observed, however, it now peaks at $s=3$. The relative intensity of this most prominent hydrogenation product is about 0.13 with respect to the precursor peak, i.e. it is significantly higher than what was observed for anthracene. Again, a broad distribution of fragments is observed albeit with more fluctuation as compared to anthracene. In particular, $\mathrm{C}_{1} \mathrm{H}_{m}$ loss (around $m / z=190)$ is found to be relatively weak.
Triphenylene $\left(\mathrm{C}_{18} \mathrm{H}_{12}^{+}, M=228\right)$

Figure 2c shows the results for the 4 ring PAH triphenylene, a peri-condensed PAH with a very open structure. Triphenylene in the gas-phase has barely been studied, despite its fully benzenoid nature, distinguishing it from most other PAHs [28]. Benzenoid PAHs are of relatively low reactivity and are particularly stable. The hydrogenation pattern is very similar as observed for pyrene, with a peak at $s=3$. However, the relative intensity of the intact hydrogenated PAH cation is about a factor of 2 higher as compared to pyrene and fragmentation is rather weak. For triphenylene, the fragmentation pattern exhibits the typical bi-modal structure with abundant smaller fragments ( $n=7-10)$, intermediate fragments being weak, and very large fragments being strong again. Particularly, $\mathrm{C}_{2} \mathrm{H}_{m}$ loss is weaker than $\mathrm{C}_{1} \mathrm{H}_{m}$ loss. This is again a surprising result as commonly $\mathrm{C}_{2} \mathrm{H}_{m}$ loss is dominating.

Tetracene $\left(\mathrm{C}_{18} \mathrm{H}_{12}^{+}, M=228\right)$

Figure 2d shows the results for the 4 ring PAH tetracene, an ortho-condensed PAH such as anthracene. Tetracene is isomeric to triphenylene but lacks the benzenoic nature. This makes it ideal for comparing the effect of PAH structure on hydrogenation and fragmentation. We observe a very similar relative intensity of the intact hydrogenated species with just a slight increase of fragmentation. The hydrogenation pattern shown in the insets is different, though. For tetracene, hydrogenation peaks at $s=1$ and decreases monotonically with $s$. The pattern is alike to what is observed for the structurally very similar anthracene. Hydrogenation up to $s=7$ is observed. This is markedly different from triphenylene, where $s=3$ dominates but $s=5$ is already very weak and $s=7$ is not observed. For tetracene, the fragmentation pattern exhibits the typical bi-modal structure as in the case of triphenylene.

\section{8-9-Benzofluoranthene $\left(\mathrm{C}_{20} \mathrm{H}_{12}^{+}, M=252\right)$}

8-9-Benzofluoranthene is a planar PAH containing a 5-membered ring. Besides PAHs, fullerenes and their cations have long been thought to play a key role in the chemistry of the interstellar medium. Fullerenes have been observed in circumstellar and nebular environments [2931 ] and very recently in diffuse ISM with a robust confirmation from the Hubble telescope [32]. A key difference between fullerenes and the PAHs discussed above is the presence of 5-membered rings in fullerenes. Berne and Tielens [33] have proposed that fullerene formation in the ISM could be possible by UV-assisted processing of PAHs that contain 5-membered rings. Figure 2e shows our hydrogenation results for 8-9-Benzofluoranthene. Even though this $\mathrm{PAH}$ is the largest under study, the relative yield of intact hydrogenated species is smaller than for triphenylene and tetracene.

$s=1$ is very weak and $s=3$ is the strongest peak in the spectrum. Peak intensities monotonically decrease 


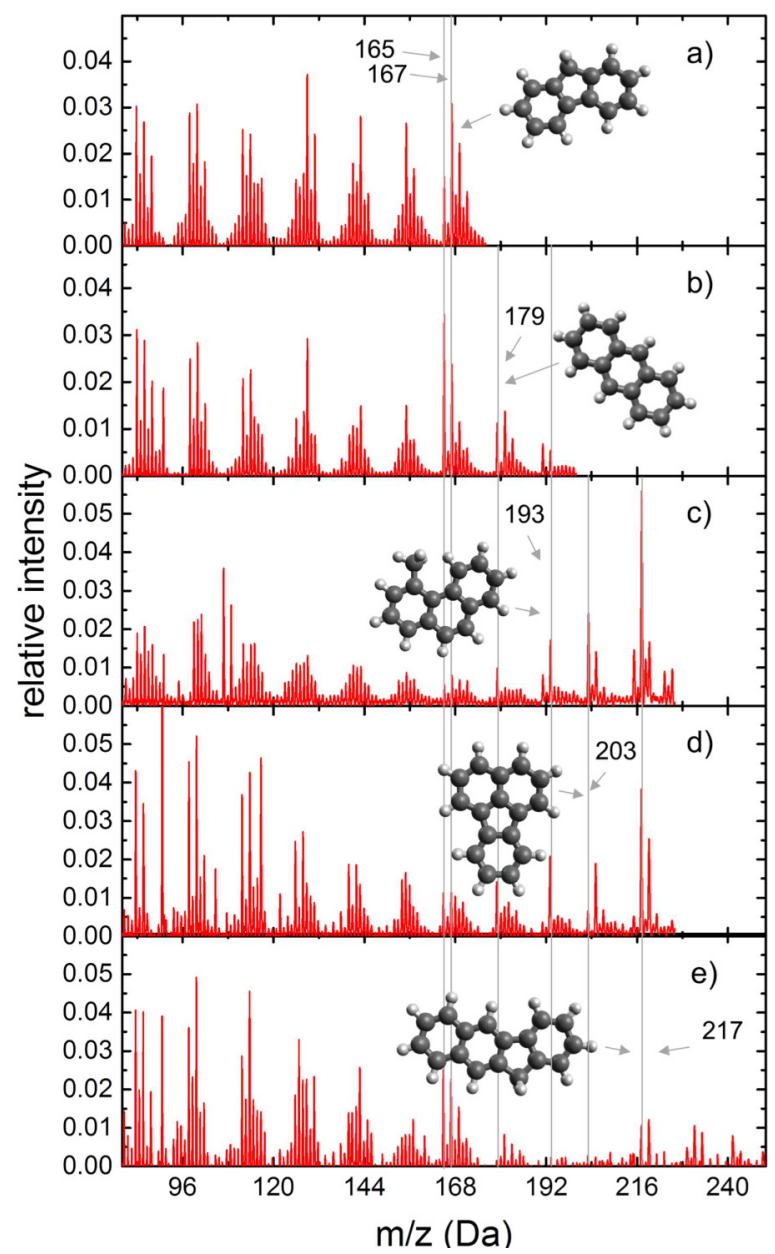

Fig. 3. Compilation of mass spectra for anthracene (a), pyrene (b), triphenylene (c), tetracene (d) and 8-9-benzofluoranthene. Only the mass range below the precursor mass is shown. The vertical solid lines indicate a number of particularly strong larger fragments. Potential geometries for the indicated masses are from top to bottom: fluorene $\left(\mathrm{C}_{13} \mathrm{H}_{10}\right)$, anthracene $\left(\mathrm{C}_{14} \mathrm{H}_{10}\right)$, methyl-phenantrene $\left(\mathrm{C}_{15} \mathrm{H}_{12}\right)$, fluoranthene $\left(\mathrm{C}_{16} \mathrm{H}_{10}\right)$ and benzofluorene $\left(\mathrm{C}_{17} \mathrm{H}_{12}\right)$.

until $s=9$ which is unambiguously observed. As every hydrogen attachment involves deposition of the respective binding energy into the system, larger average numbers of attached $\mathrm{H}$ atoms $s$ imply higher excitation energies and the systems is thus more prone to fragmentation.

\subsection{Fragmentation as a function of PAHs size}

Our experiments focus on the hydrogenation of PAHs of different sizes as well as their resulting fragmentation. Successive attachment of $\mathrm{H}$ atoms is accompanied by deposition of the respective binding energy. As a consequence, in the course of the hydrogenation sequences PAH cations heat up. At the same time, the successive transition from an aromatic planar molecule to an aliphatic system is accompanied by an overall decrease in stability. Substantial fragmentation is already observed at very moder- ate hydrogenation states, where structural weakening is not yet expected to play an important role. We therefore conclude that fragmentation is mostly due to excess (thermal) excitation energy that cannot be dissipated sufficiently fast (e.g. by photon emission) to prevent molecular breakup. As fragment ions remain trapped for the rest of each exposure cycle, there is a small probability that they are subjected to hydrogenation which may affect the fragmentation pattern.

Furthermore, the fragmentation pattern appears to be different for 3 or 4 rings PAHs and depending on whether or not the phenyl rings share common carbon atoms. There is a clear distinction between a bimodal fragmentation pattern, as seen in Figures 2d, 2e and a flat fragmentation pattern as seen in Figure 2a. A zoom into the fragmentation patterns is shown in Figure 3.

In a previous work, we simulated the fragmentation patterns according to different assumptions on the cooling efficiencies [20]. In this framework, the flat fragmentation distribution can be explained by assuming a total absence of radiative cooling (incremental scenario; internal energy corresponds to $s$ successive attachments of atomic hydrogen). This implies that the internal PAH temperature is so high that fragmentation sets in before the temperature could decrease appreciably. A flat fragmentation pattern can also be obtained in a model where PAHs are thermalized by a $3000-3500 \mathrm{~K}$ heat bath (Boltzmann scenario). Also in this case, fragmentation proceeds at very high internal temperatures and cooling is only active for even higher internal temperatures. It is straightforward that this scenario is most closely met experimentally for anthracene, the smallest PAH under study which is subject to the highest internal temperature increase upon $\mathrm{H}$ attachment.

The bimodal distribution on the other hand is based on the assumption of efficient cooling. In the model, this is implemented by broadening the molecular internal energy distribution with respect to a Boltzmann distribution (Broad scenario). Applied to our experimental findings, we can deduce cooling processes are (i) stronger for PAHs with 4 rings than for those with 3 rings (ii) stronger for independent phenyl rings than peri-condensed PAHs.

Figure 3 also allows a closer inspection of the fragment masses. A number of the most prominent larger fragments are marked with vertical lines. All indicated peaks can be assigned to closed shell (hydrogenated or dehydrogenated) versions of the different PAHs. For instance, for anthracene (a) we observe strong peaks at 165 and 167 , which could be assigned to fluorene $\left(\mathrm{C}_{13} \mathrm{H}_{10}\right)$ which can be formed from anthracene by removal of a single $\mathrm{C}$ atom from the center ring. Interestingly, these peaks are even most prominent for pyrene (b). In (b), there is also a strong peak at 179 that is also prominent in (c) and (d). This peak can be assigned to phenantrene (b) and (c) or anthracene (d). A peak at 193, present in (b)-(d) can be assigned to various isomers of methyl-phenantrene (shown as an inset) or methyl-anthracene $\left(\mathrm{C}_{15} \mathrm{H}_{12}\right)$. The peak at 203 observed in (c)-(e) could be due to pyrene which is unlikely formed from these 3 precursors. For $(\mathrm{c}, \mathrm{e})$ this peak is most likely due to fluoranthene $\left(\mathrm{C}_{16} \mathrm{H}_{10}\right)$ (shown as inset). Last but not least, (c)-(e) feature a strong peak 
at 217 that could be due to the benzofluorene family of structures $\left(\mathrm{C}_{17} \mathrm{H}_{12}\right)$. A number of fragment cations feature 5-membered rings. This is of particular interest for fullerene formation.

\section{3 $\mathrm{H}_{2}$ abstraction}

As discussed in the introduction, $\mathrm{H}$ attachment competes with Eley-Rideal-type abstraction reactions (see Eq. (2) and [14]). In the previous section, we have shown that multiple hydrogen attachment is a generic process that is observed on all smaller PAH cations under study. It is very difficult however, to find evidence for abstraction processes in the presented data. As in our recent study on coronene [14], we have therefore used a thermal $\mathrm{D}$ beam to investigate hydrogen abstraction in pyrene cations $\mathrm{C}_{16} \mathrm{H}_{10}^{+}$. The following scheme shows the possible reactions with $r$ indicating the number of attached D atoms and $q$ indicating the number of abstracted $\mathrm{H}$ atoms.

$$
\mathrm{C}_{16} \mathrm{H}_{(10-q)} \mathrm{D}_{r}^{+}+\mathrm{D} \longrightarrow\left\{\begin{array}{l}
\mathrm{C}_{16} \mathrm{H}_{(10-q)} \mathrm{D}_{(r+1)}^{+} \\
\mathrm{C}_{16} \mathrm{H}_{(10-q)} \mathrm{D}_{(r-1)}^{+}+\mathrm{D}_{2} \\
\mathrm{C}_{16} \mathrm{H}_{(10-q-1)} \mathrm{D}_{r}^{+}+\mathrm{HD}
\end{array}\right.
$$

For both attachment and abstraction of $\mathrm{D}$ (the first 2 reactions) $|\Delta M|=2$ and as for a thermal $\mathrm{H}$ beam, no evidence for abstraction can be extracted. It is reaction 3 associated to HD abstraction that leads to formation of additional peaks in the mass spectrum with odd $\Delta M$, that are evidence for abstraction. Figure 4 shows the obtained mass spectra for various exposure times as a function of $\Delta M$, i.e. the mass difference relative to the mass of the precursor ion. Clearly, already for $t_{\exp }=0.15 \mathrm{~s}$ almost all precursor ions have been singly deuterated and the peak at $\Delta M=2$ dominates. After $1 \mathrm{~s}$, the precursor ion at $\Delta M=0$ is almost fully depleted. We do not observe the abstraction reactions

$$
\mathrm{C}_{16} \mathrm{H}_{10}^{+}+\mathrm{D} \longrightarrow \mathrm{C}_{16} \mathrm{H}_{9}^{+}+\mathrm{HD}
$$

and

$$
\mathrm{C}_{16} \mathrm{H}_{10} \mathrm{D}^{+}+\mathrm{D} \longrightarrow \mathrm{C}_{16} \mathrm{H}_{9} \mathrm{D}^{+}+\mathrm{HD}
$$

as they would manifest in peaks at $\Delta M=-1$ and $\Delta M=1$, respectively, which are absent in all spectra.

It can be seen from Figure 4 that already for $t_{\exp }=0.5 \mathrm{~s}$, first indications for abstraction reactions become apparent in the form of peaks at $\Delta M=4$ and 5 (The peak at $\Delta M=3$ is in part due to the natural abundance of ${ }^{13} \mathrm{C}$, present in the precursor ions. The peak at $\Delta M=4$ could also be due to attachment of $2 \mathrm{D}$ atoms, a channel that is expected to be weak). With increasing $t_{\exp }$, these fingerprints of HD formation exhibit the expected increase in intensity and for $t_{\exp }=6.0 \mathrm{~s}$ we observe a mass spectrum very similar to the one for coronene cations [14] (Although we only display the mass range close to the precursor mass in Fig. 4 , particularly at $t_{\exp }=3$ and $6 \mathrm{~s}$, substantial fragmentation is observed as well.) Peaks mostly due to attachment of 1,3 and $5 \mathrm{D}$ atoms to $\mathrm{C}_{12} \mathrm{H}_{10}^{+}$ can be observed at $\Delta M=2,6$ and 10 respectively. Peaks

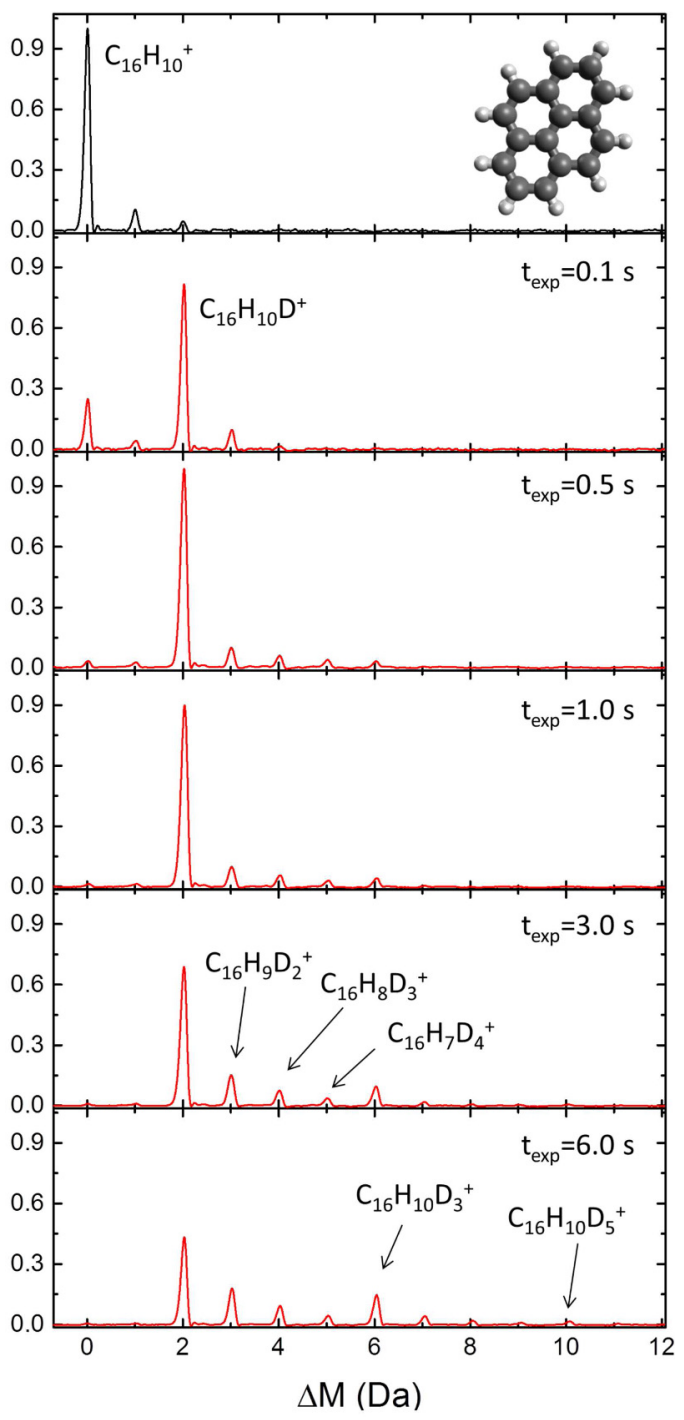

Fig. 4. Mass spectra of pyrene for the precursor cations (top) and after exposure to atomic deuterium for $t_{\exp }=0.1 \mathrm{~s}$ increasing to $t_{\exp }=6 \mathrm{~s}$ (bottom). The mass is given in $\Delta M$, relative to the mass of the precursor cation.

due to abstraction reactions involving HD mostly give rise to peaks at $\Delta M=3,4$ and 5 and again at $\Delta M=7,8$ and 9. It is thus clear that the interplay of hydrogen attachment and hydrogen abstraction is not limited to coronene cations but can be observed in a very similar fashion for the pyrene cation. Most likely, abstraction reactions have to be considered for most PAH cations.

\section{Conclusions}

Hydrogen attachment to and hydrogen abstraction by Eley-Rideal reactions from gas-phase coronene cations had been investigated in detail, previously. Here, we have shown that attachment occurs also on a series of different smaller PAH cations where it gives rise to similar hydrogenation patterns. Abstraction was investigated for the 
pyrene cation, where it proceeds in a similar fashion previously observed for coronene. Our experimental findings therefore suggest that very likely both processes are common for gas-phase PAH cations. This has important astrochemical implications as hydrogenation attachment and abstraction should accordingly be included in models of $\mathrm{PAH}$ growth and $\mathrm{H}_{2}$ formation. Note that in many astrochemical environments, sequential hydrogenation will occur on time-scales that are orders of magnitude larger than the time-scales investigated here. As a consequence, energy completely dissipates between $\mathrm{H}$ attachment events, quenching hydrogenation-induced fragmentation.

As hydrogen attachment events are accompanied by deposition of the binding energy into the molecular cation, sequences of such events lead to substantial heating, that competes with cooling by photon emission. From the hydrogen attachment induced fragmentation patterns, we can deduce that cooling is strongly dependent on the PAH structure. Cooling seems to be stronger for PAHs with 4 rings than for those with 3 rings and also stronger for independent phenyl rings than peri-condensed PAHs.

\section{Author contribution statement}

All the authors were involved in the preparation of the manuscript. All the authors have read and approved the final manuscript.

Publisher's Note The EPJ Publishers remain neutral with regard to jurisdictional claims in published maps and institutional affiliations.

Open Access This is an open access article distributed under the terms of the Creative Commons Attribution License (https://creativecommons.org/licenses/by/4.0/), which permits unrestricted use, distribution, and reproduction in any medium, provided the original work is properly cited.

\section{References}

1. A. Leger, J.L. Puget, Astron. Astrophys. 137, L5 (1984)

2. L.J. Allamandola, A.G.G.M. Tielens, J.R. Barker, Astrophys. J. 290, L25 (1985)

3. C.W. Bauschlicher, Astrophys. J. 509, L125 (1998)

4. J. Oomens, B.G. Sartakov, A.G.G.M. Tielens, G. Meijer, G. von Helden, Astrophys. J. Lett. 560, L99 (2001)

5. A.G.G.M. Tielens, Interstellar PAHs and dust, in Planets, Stars and Stellar Systems, edited by T.D. Oswald, G. Gilmore (Springer, Doordrecht, 2013), pp. 499-548

6. T.P. Snow, V. Le Page, Y. Keheyan, V.M. Bierbaum, Nature 391, 259 (1998)

7. E. Rauls, L. Hornekær, Astrophys. J. 679, 531 (2008)

8. J.D. Thrower, B. Jørgensen, E.E. Friis, S. Baouche, V. Mennella, A.C. Luntz, M. Andersen, B. Hammer, L. Hornekær, Astrophys. J. 752, 3 (2012)

9. L. Boschman, G. Reitsma, S. Cazaux, T. Schlatholter, R. Hoekstra, M. Spaans, O. Gonzalez-Magana, Astrophys. J. Lett. 761, L33 (2012)
10. S. Cazaux, L. Boschman, N. Rougeau, G. Reitsma, R. Hoekstra, D. Teillet-Billy, S. Morisset, M. Spaans, T. Schlathölter, Sci. Rep. 6, 19835 (2016)

11. S. Cazaux, Y. Arribard, D. Egorov, J. Palotas, R. Hoekstra, G. Berden, J. Oomens, T. Schlathölter, Astrophys. J. 875, 7 (2019)

12. P.A. Jensen, M. Leccese, F.D.S. Simonsen, A.W. Skov, M. Bonfanti, J.D. Thrower, R. Martinazzo, L. Hornekær, Mon. Not. R. Astron. Soc. 486, 5492 (2019)

13. V. Mennella, L. Hornekær, J. Thrower, M. Accolla, Astrophys. J. Lett. 745, L2 (2012)

14. N. Foley, S. Cazaux, D. Egorov, L.M.P.V. Boschman, R. Hoekstra, T. Schlathölter, Mon. Not. R. Astron. Soc. 479, 649 (2018)

15. D. Campisi, F.D.S. Simonsen, J.D. Thrower, R. Jaganathan, L. Hornekr, R. Martinazzo, A.G.G.M. Tielens, Phys. Chem. Chem. Phys. 22, 1557 (2020)

16. L. Boschman, S. Cazaux, M. Spaans, R. Hoekstra, T. Schlathölter, Astron. Astrophys. 579, A72 (2015)

17. R. Hoekstra, F.J. de Heer, R. Morgenstern, J. Phys. B: At. Mol. Opt. Phys. 24, 4025 (1991)

18. G. Reitsma, L. Boschman, M.J. Deuzeman, O. GonzalezMagana, S. Hoekstra, S. Cazaux, R. Hoekstra, T. Schlathölter, Phys. Rev. Lett. 113, 053002 (2014)

19. A. Castellanos, P. Benigni, D.R. Hernandez, J.D. DeBord, M.E. Ridgeway, M.A. Park, F. Fernandez-Lima, Anal. Methods 6, 9328 (2014)

20. M. Rapacioli, S. Cazaux, N. Foley, A. Simon, R. Hoekstra, T. Schlatholter, Phys. Chem. Chem. Phys. 20, 22427 (2018)

21. H.W. Jochims, E. Rühl, H. Baumgärtel, S. Tobita, S. Leach, Astrophys. J. 420, 307 (1994)

22. R Arakawa, M. Kobayashi, T. Nishimura, J. Mass Spectrom. 35, 178 (2000)

23. P.J.M. van der Burgt, M. Dunne, M.L. Gradziel, Eur. Phys. J. D 72, 31 (2018)

24. J. Postma, S. Bari, R. Hoekstra, A.G.G.M. Tielens, T. Schlathölter, Astrophys. J. 708, 435 (2010)

25. P. Rousseau, A. Lawicki, A.I.S. Holm, M. Capron, R. Maisonny, S. Maclot, E. Lattouf, H.A.B. Johansson, F. Seitz, A. Mery, J. Rangama, H. Zettergren, S. Rosen, H.T. Schmidt, J.Y. Chesnel, A. Domaracka, B. Manil, L. Adoui, H. Cederquist, B.A. Huber, Nucl. Instrum. Methods Phys. Res. Sect. B 279, 140 (2012)

26. A.I.S. Holm, H.A.B. Johansson, H. Cederquist, H. Zettergren, J. Chem. Phys. 134, 044301 (2011)

27. M.H. Stockett, M. Gatchell, T. Chen, N. de Ruette, L. Giacomozzi, M. Wolf, H.T. Schmidt, H. Zettergren, H. Cederquist, J. Phys. Chem. Lett. 6, 4504 (2015)

28. V. Kofman, P.J. Sarre, R.E. Hibbins, I.L. ten Kate, H. Linnartz, Mol. Astrophys. 7, 19 (2017)

29. J. Cami, J. Bernard-Salas, E. Peeters, S.E. Malek, Science 329, 1180 (2010)

30. K. Sellgren, M.W. Werner, J.G. Ingalls, J.D.T. Smith, T.M. Carleton, C. Joblin, Astrophys. J. Lett. 722, L54 (2010)

31. O. Berné, G. Mulas, C. Joblin, Astron. Astrophys. 550, L4 (2013)

32. M.A. Cordiner, H. Linnartz, N.L.J. Cox, J. Cami, F. Najarro, C.R. Proffitt, R. Lallement, P. Ehrenfreund, B.H. Foing, T.R. Gull, P.J. Sarre, S.B. Charnley, Astrophys. J. Lett. 875, L28 (2019)

33. O. Berne, A.G.G.M. Tielens, Proc. Natl. Acad. Sci. U.S.A. 109, 401 (2012) 